\title{
Physiological and transcriptomic analyses of the effects of S/BRI1 expression levels on drought tolerance in tomato seedlings
}

\section{Shuming Nie}

China West Normal University

\section{Zaijun Yang}

China West Normal University

Dan Wang ( $\square$ wangdd0310@163.com )

China West Normal University

\section{Research Article}

Keywords: SLBRI1, Tomato, Drought, Physiology, Transcriptome

Posted Date: February 17th, 2021

DOI: https://doi.org/10.21203/rs.3.rs-200334/v1

License: (c) (i) This work is licensed under a Creative Commons Attribution 4.0 International License.

Read Full License

Version of Record: A version of this preprint was published at Journal of Plant Growth Regulation on February 4th, 2022. See the published version at https://doi.org/10.1007/s00344-022-10587-4. 


\section{Abstract}

Background: Brassinosteroids (BRs) not only influence plant growth and development but also regulate the various stress responses of plants. BRASSINOSTEROID-INSENSITIVE 1 (BRI1) acts as a BR receptor, sensing the BRs and then activating BR signaling. In this study, how SIBR/1 regulate the drought resistance of tomato have further been researched at physiological and transcriptomic level.

Results: We obtained S/BR/1-overexpressing and SIBR/1 weak mutant (abs) plants in the same background to research the underlying mechanism in drought resistance. In this study, physiological analyses revealed that abs plants had a higher net photosynthetic rate and less wilting than MM plants; abs plants also had lower $\mathrm{H}_{2} \mathrm{O}_{2}$ and $\mathrm{O}_{2}{ }^{-}$accumulation through higher antioxidant enzyme activities under drought conditions. RNA-Seq analysis showed that 768 (53.9\%) of 1425 drought-induced genes and $418(49.8 \%)$ of 840 drought-repressed genes were regulated by abs in the same direction under normal conditions. Moreover, 158 drought-induced and 43 drought-repressed genes are further upregulated and downregulated in abs plants under drought conditions, respectively. An in-depth analysis of these DEGs revealed that abs regulated the expression of genes related to ABA metabolism and polyamine biosynthesis as well as oxidoreductase activity genes under normal and drought conditions. Furthermore, analysis of transcription factor expression suggested that abs affected drought tolerance mainly through the regulation of WRKY, ERF, bHLH and MYB transcription factors. However, the expression of most of these genes was the same or opposite in SIBRI1OE plants compared to the MM group.

Conclusion: Our results establish that SIBR/1 expression level was negatively involved in drought responses of tomato. Furthermore, our study provides valuable information for future breeding to appropriately reduce the expression of S/BR/1 and improve drought resistance without affecting plant growth.

\section{Background}

Water availability is one of the most important environmental factors limiting plant species distribution and agricultural production [1]. When a plant suffers mild water stress, its photosynthesis and respiration rates decrease, and a series of physiological and biochemical responses also change to adapt to the drought stress [2]. More severe water stress can inhibit growth and damage the ultrastructures of cells and organelles, which results in cellular dehydration, the accumulation of toxic substances, the loss of cell membrane permeability, the inactivation of enzymes, and changes in protein structure. All of these factors eventually lead to metabolic disturbances and even the death of the plant [3-6]. Furthermore, drought stress can also induce the enhanced production of reactive oxygen species (ROS), which leads to oxidative stress [7]. To resist drought-induced oxidative stress, plants can eliminate excess ROS by upregulating the activity of enzymatic and nonenzymatic antioxidants [8]. Increased antioxidant enzyme activities play an important role in plant drought resistance [9]. 
Brassinosteroids (BRs) are steroid hormones that exist in all plant tissues. BRs play important roles in plant growth and development processes, such as promoting seed germination, cell elongation and division, pollen fertility, vascular differentiation, fruit set, and seed setting rate [10-15]. In addition, BRs can enhance plant photosynthesis and chlorophyll content, delay ageing, and elevate stress resistance[16]. To date, well-developed BR signal transduction models have been established in Arabidopsis. BRs first bind to the plasma membrane-localized receptor kinase BRASSINOSTEROID INSENSITIVE1 (BRI1) and the coreceptor BRI1-ASSOCIATED RECEPTOR KINASE1 (BAK1) [17-20]. BRI1 interacts with BAK1 and activates BR signalling, after which the BR signals are transduced to downstream components. Finally, dephosphorylated BES1 (bri1-EMS-suppressor 1) and BZR1 (Brassinazole-resistant 1) accumulate in the nucleus to regulate the expression of thousands of $B R$ response genes $[21,22]$.

Brassinosteroid (BR) signalling intensity not only influences plant growth and development but also regulates the various stress responses of plants. OsGSK1 (Oryza sativa glycogen synthase kinase 3-like gene 1) is an orthologue of Arabidopsis brassinosteroid insensitive 2 (BIN2), and knockout of OsGSK1 enhances tolerance to cold, heat, salt, and drought stresses[23]. The Arabidopsis elongated-D mutant, a BAK1 single base gain-of-function mutant, increases BR signalling and is more vulnerable to a bacterial pathogen and salinity stress[24]. The Arabidopsis transcription factor RESPONSIVE TO DESICCATION26 (RD26) inhibits BR-regulated growth but increases drought tolerance by upregulating the expression of drought-induced genes. RD26 mediates crosstalk between drought and BR signalling[25]. WRKY46, WRKY54, and WRKY70 positively regulate BR signalling and plant growth, while WRKYs negatively regulate drought tolerance by inhibiting dehydration-inducible gene expression[26]. The AP2/ERF transcription factor TINY negatively regulates plant growth and compromises BR-responsive gene expression. TINY positively regulates drought resistance by promoting drought-responsive gene expression[27]. Wheat brassinazole-resistant 2 (TaBZR2) positively regulates BR signalling and drought responses. TaBZR2 directly activates the expression of the $T$. aestivum glutathione-transferase-1 (TaGST1) gene, which can scavenge drought-induced superoxide anions[28].

The expression level of the BR receptor BRI1 directly influences BR signal intensity and drought resistance. RNAi of a BRI1 homologue in Brachypodium distachyon decreases the growth potential of plants while increasing their tolerance to drought and drought-responsive gene expression[29]. Arabidopsis gain-of-function BR mutants (bes1-D) negatively regulate drought tolerance by repressing the expression of drought response genes, while the BR weak mutant (bri1-5) increases drought tolerance by upregulating the expression of these genes [25]. Overexpression of the vascular BR receptor BRL3 increases drought tolerance and the accumulation of osmoprotectant metabolites without affecting plant growth[30].

In a previous study, we used SIBR/1 overexpressing plants of the tomato cultivar Micro-Tom to increase BR signalling and decrease drought resistance[31]. We further found that S/BR/1 weak mutants in the tomato cultivar Money Maker (MM) had altered brassinolide sensitivity (abs) with a missense mutation in the kinase domain, delayed growth and enhanced drought resistance[31, 32]. To further understand the 
relationship between the S/BR/1 expression level and drought resistance, we obtained S/BR/1 overexpressing and SIBR/1 weak mutant (abs) plants from the same background to research the underlying mechanism in drought resistance. Recently, various advanced RNA-Seq methods have been applied in numerous organisms, enabling this technique to be widely used in identifying key stress metabolism pathways (Lu et al., 2014; Wang et al., 2009). Therefore, RNA-Seq was used to analyse the effects of different SIBR/1 expression levels on drought tolerance in tomato.

\section{Results}

\section{Physiological analysis}

\section{SIBRI1 transgenic plants have increased SIBRI1 expression levels}

To investigate the associations of drought tolerance with the SIBR/1 expression level, we generated transgenic tomato plants in the tomato cultivar 'Money Maker' background in which $S / B R / 1$, driven by the constitutive CaMV 35S promoter, was overexpressed. The transcript levels of $S / B R / 1$ in the two transgenic lines, SIBR/1-OE-6 and SIBR/1-OE-7, were 5.4 and 16.5 times higher, respectively, than those in MM plants (Fig. 1B). Moreover, the SIBR/1 protein levels in the SIBR/1-OE-6 and SIBR/1-OE-7lines were confirmed by Western blot analysis (Fig. 1A). The expression levels of the BR biosynthetic genes DWARF and CPD were significantly lower than those in MM plants (Fig. 1C and D). These results showed that transgenic plants had increased SIBR/1 expression levels and BR signaling intensity.

\section{SIBRI1 expression level alters plant growth, leaf relative water content and electrolyte leakage under drought stress}

To investigate whether the $S / B R / 1$ expression level is related to the drought resistance of tomato seedlings, SIBR/1 overexpressing, MM and abs (SIBR/1 weak mutants) tomato seedlings were all subjected to drought stress (water withheld) for $12 \mathrm{~d}$. After $12 \mathrm{~d}$ of drought stress, all plants showed different degrees of leaf wilting. The abs plants showed slight wilting, while the wilting was the most serious in the SIBR/1-OE-6 and SIBR/1-OE-7 plants (Fig. 2A, B).

Drought stress decreased leaf relative water content (RWC), and the leaf RWC in SIBR/1 overexpressing lines was significantly lower than that in MM plants, while the RWC of abs plants was significantly higher than that of MM plants at $10 \mathrm{~d}$ of drought stress, and was $24.5 \%, 30.6 \%$ and $35.6 \%$ higher than those of MM, SIBRI1-OE-6 and SIBR/1-OE-7 plants, respectively (Fig. 2C). Drought stress resulted in enhanced electrolyte leakage levels in all plants. The leaf electrolyte leakage levels of $S / B R / 1$ overexpressing lines were significantly higher than those of MM plants, while the electrolyte leakage levels of abs plants were significantly lower than those of MM plants at $10 \mathrm{~d}$ of drought stress and were $19.1 \%, 33.6 \%$ and $40.5 \%$ lower than those of MM, SIBR/1-OE-6 and SIBR/1-OE-7 plants, respectively (Fig. 2D). These results indicated that $S / B R / 1$ expression levels negatively regulated the drought tolerance of tomato seedlings.

\section{SIBR/1 expression level affects gas exchange under drought stress}


Compared with the control, SIBR/1 overexpressing plants had slightly increased net photosynthetic rate $(\mathrm{Pn})$ and stomatal conductance (Gs) before drought stress. However, drought stress obviously decreased the $\mathrm{Pn}, \mathrm{Gs}$ and transpiration rate ( $\mathrm{Tr}$ ) but increased the intercellular $\mathrm{CO}_{2}$ concentration ( $\mathrm{Ci}$ ) in all plants (Fig. 3A-D). At $10 \mathrm{~d}$ of drought stress, the Pn and Tr of abs plants were significantly higher, and Ci was obviously lower, than those of other plants, while in SIBR/1 overexpressing plants, $\mathrm{Pn}$ and $\operatorname{Tr}$ were obviously lower, and Ci was markedly higher, than in other plants (Fig. 3A-D). For example, the Pn of abs plants was $27.1 \%, 49.2 \%$ and $56.5 \%$ higher than those of MM, SIBR/1-OE-6 and SIBR/1-OE-7 plants, respectively. There were no significant differences in Gs among all plants (Fig. 3A).

\section{S/BRI1 expression level affects accumulation of $\mathrm{H}_{2} \mathrm{O}_{2}, \mathrm{O}_{2}{ }^{-}$and antioxidant enzyme activities under drought stress}

Histochemical observations were used to assess the accumulation of hydrogen peroxide $\left(\mathrm{H}_{2} \mathrm{O}_{2}\right)$ and superoxide $\left(\mathrm{O}_{2}^{-}\right)$in tomato leaves. Before drought stress, there were no significant differences in $\mathrm{H}_{2} \mathrm{O}_{2}$ and $\mathrm{O}_{2}{ }^{-}$accumulation among all plants (Fig. 4A, B). After $10 \mathrm{~d}$ of drought stress, the accumulation of $\mathrm{H}_{2} \mathrm{O}_{2}$ and $\mathrm{O}_{2}{ }^{-}$in abs leaves was lower than that in MM leaves, but SIBR/1-overexpressing leaves accumulated much higher levels than MM (Fig. 4A, B). Therefore, abs plants had lower ROS levels, and SIBRI1 overexpressing plants had higher ROS levels than MM plants under drought stress.

Drought stress obviously increased the antioxidant enzyme activities of all plants. The SOD, POD and CAT activities of abs plants were significantly higher than those of other plants at $10 \mathrm{~d}$ of drought stress and were $39.9 \%, 47.9 \%$ and $29.8 \%$ higher than those of SIBR/1-OE-6 plants, respectively. The APX activities of $\mathrm{MM}$ and abs plants were significantly higher than those of S/BR/1 overexpressing plants after drought stress (Fig. 4C-F). These results showed that BR signalling was negatively related to the antioxidant enzyme activities of tomato seedlings under drought stress.

\section{Transcriptome Analysis}

\section{Sequencing of different SIBRI1 expression level plants RNA-Seq}

To further understand how SIBR/1 negatively regulates drought responses, we performed global gene expression studies with MM, SIBR/1-OE-7 and abs plants by high-throughput RNA sequencing (RNA-seq). As shown in Table 1, six cDNA libraries (three replicates per library) were constructed. RNA-Seq of these libraries generated approximately 47-62 million total reads, with an average of $98.9 \%$ clean reads obtained after quality filtering. Clean reads from every library showed a match rate of approximately $95 \%$ to the tomato genome, indicating that the sequencing data could be used for subsequent transcriptome analysis (Table 1).

Table 1

Summary of RNA-Seq datasets. 


\begin{tabular}{|c|c|c|c|c|c|}
\hline Sample & Total reads & Clean reads & Multiple mapped & Uniquely mapped & Q30(\%) \\
\hline MM_1 & 234 & 49000446 & $1682647(3.43 \%)$ & $45330385(92.51 \%)$ & 95.14 \\
\hline MM & 51088508 & 50546354 & $2757649(5.46 \%)$ & $44715920(88.47 \%)$ & 95.33 \\
\hline $\mathrm{MM}^{-} 3$ & 88844 & 47336100 & $3387809(7.16 \%)$ & $38838001(82.05 \%)$ & 95.45 \\
\hline$S I B \bar{R} I O E \_1$ & 028 & 51003574 & $1536162(3.01 \%)$ & $47370570(92.88 \%)$ & 10 \\
\hline SIBRIIOE 2 & & 3380 & & $\%$ & \\
\hline SIBRIIOE-3 & & & 20 & $6 \%)$ & \\
\hline & & 4982 & & & \\
\hline & & 986 & 23 & 5 & \\
\hline & & 982 & $9(3.62 \%)$ & $(92.65 \%)$ & \\
\hline MMĪ & & 80 & $3(2.8$ & $4(9$ & 5.35 \\
\hline & & & $663(3.46 \%)$ & $200(93.22 \%)$ & 95.34 \\
\hline MMD & & 08 & & $200(00.24 \pi$ & 95.59 \\
\hline SIBRIIOE DS 1 & & & $4 \%$ & $2.87 \%)$ & \\
\hline SIBRI1OE $\mathrm{DS}^{-1}$ & & & 13 & $4.27 \%)$ & \\
\hline SIBRI1OE $\mathrm{DS}^{-} 3$ & & & 25 & $8.35 \%)$ & 95.27 \\
\hline absDS 1 & & 1416 & $288-1-1-1$ & $020(91.75 \%)$ & 95.11 \\
\hline$a b s$ & & 62142522 & 194 & $26086(90.96 \%)$ & \\
\hline$a b s D S 3$ & 10 & 58470256 & $2040186(3.49 \%)$ & $53175772(90.94 \%)$ & or. \\
\hline
\end{tabular}

\section{SIBRI1 negatively regulates drought responsive genes expression}

Analysis of DEGs was performed between every pair of groups (MMDS/SIBR/1OEDS, MMDS/absDS, $\mathrm{MM} / a b s$, abs/absDS, MM/MMDS, SIBR/1OE/SIBR/1OEDS and MM/SIBR/1OE) based on FPKM with thresholds FDR $<0.05$ and FC $>2$. There were 3144 DEGs (2096 up- and 1048 downregulated) between MMDS and absDS, 6162 DEGs (3873 up- and 2289 downregulated) between MM and abs, and 2265 DEGs (1425 up- and 840 downregulated) between MM and MMDS. There were only 85 DEGs between MM and SIBR/1OE, comprising 59 upregulated and 26 downregulated DEGs (Fig. 5). We selected the above 4 groups for comprehensive analysis. The number of upregulated DEGs was higher than that of downregulated DEGs across all four comparisons (Fig. 5). However, we further found that 768 (53.9\%) of 1425 drought-induced and 418 (49.8\%) of 840 drought-repressed genes were regulated by abs in the same direction under normal conditions (Fig. 6A, B). There were 158 drought-induced and 43 droughtrepressed genes that were further upregulated and downregulated in $a b s \mathrm{DS}$, respectively (Fig. 6A, B). These results indicated that abs may regulate the expression of a number of drought-related genes, which results in the inhibition of plant growth under normal conditions, consistent with the growth phenotype of abs.

To further investigate how SIBR/1-OE and abs affect drought-related gene expression, we performed clustering analysis of the genes that were upregulated and downregulated in each treatment. Under normal conditions, MM upregulated genes and downregulated genes were repressed and induced under drought conditions, respectively (Fig. 6C). However, many drought stress-induced genes and repressed genes were already upregulated and downregulated in abs under normal conditions, respectively (cluster b). Many drought stress-induced genes had higher expression in absDS, while many drought stressrepressed genes had lower expression in absDS under drought conditions (cluster a). Overall, our transcriptome analyses support a role of the SIBR/1 expression level in modulating drought-responsive gene expression, largely in an antagonistic manner. 


\section{Gene Ontology (GO) and GO enrichment analyses of different SIBR/1 levels under normal conditions}

To explore the effects of $S / B R / 1$ overexpression under normal conditions, we performed GO analyses, focusing on differences between the MM group and the SIBR/1OE group. There were 71 DEGs, 72 DEGs and 74 DEGs enriched in "biological process", "cellular component" and "molecular function", respectively (Fig. 7, Table S2). The main biological process categories were "metabolic process" and "cellular process". DEGs in the molecular function category were related to "catalytic activity" and "binding". The most DEGs were assigned to the "cell" and "membrane part" cellular component categories (Fig. 7, Table S2). These results highlighted the involvement of SIBRI1-OE in cellular processes and metabolic processes, consistent with the regulation of growth by SIBRI1-OE under normal conditions.

To explore how abs functions under normal conditions, we performed GO enrichment analyses, focusing on differences between the MM group and abs group. The top 20 most obviously enriched pathways are shown in Fig. 8. The DEGs were enriched for "photosynthesis, light harvesting in photosystem I", "photosynthesis, light harvesting", "protein phosphorylation", "phosphorylation”, "plastoglobule", "cellular protein modification process", "protein modification process", "response to abiotic stimulus", "lipid metabolic process", "phosphorus metabolic process", "phosphate-containing compound metabolic process", "carbohydrate metabolic process" and "intrinsic component of membrane" (Fig. 8, Table S3). In addition, the most enriched category for DEGs in this study was "catalytic activity", with a total of 2445 DEGs. A total of 148 DEGs were annotated as "response to abiotic stimulus" (Fig. 8, Table S3). These results indicated that abs upregulated the expression of a number of stress response-related genes under normal conditions.

\section{Differences in stress response gene expression between different treatments}

To further explore how abs increased drought resistance, we selected stress-related metabolic pathways from $\mathrm{GO}$ enrichment analyses. Four ABA biosynthesis genes, 9-cis-epoxycarotenoid dioxygenase 2 (Solyc08g016720.1), notabilis 9-cis-epoxycarotenoid dioxygenase (Solyc07g056570.1), beta-carotene, Pfam: PF05834 (Solyc06g074240.3), and zeaxanthin epoxidase (Solyc02g090890.4), were upregulated in MM under drought stress (Table S4). These genes were also induced in abs compared with MM under normal conditions. However, the expression of these genes was not obviously changed in S/BR/1OE plants compared with MM plants. Solyc08g016720.1 and Solyc06g074240.3 were upregulated between MMDS and absDS (Table S4). Eleven of 13 polyamine biosynthetic processes were upregulated in MM under drought stress. All 13 genes were induced in abs compared with MM under normal conditions. However, the expression of these genes was not obviously changed in S/BR/1OE plants compared with MM plants. Eight of 13 were upregulated between MMDS and absDS. These results indicated that $a b s$ may increase $A B A$ and polyamine contents by inducing $A B A$ and polyamine biosynthetic gene expression under normal and drought conditions (Table S5). Seven of 11 oxidoreductase activity genes were upregulated in MM under drought stress. All 11 genes were induced in abs compared with MM under normal conditions. However, the expression of most of these genes was not obviously changed in SIBR/1OE plants compared with MM plants. Nine of 11 genes were upregulated between MMDS and 
absDS, whereas 2 of 11 genes were downregulated (Fig. 9A; Table S6). These results indicated that abs may decrease reactive oxygen content by inducing oxidoreductase activity gene expression and further enhance antioxidant enzyme activities under normal and drought conditions.

Transcription factors (TFs) play a critical role in abiotic stress via gene regulatory networks. We further selected 25 TFs in 7 different families from the MMDS group, 18 upregulated and 7 downregulated. Most of the differentially expressed TFs participate in the drought stress response, and the majority are derived from the WRKY, ERF, bHLH and MYB families (Fig. 9B; Table S7). Eighteen drought stress-induced TFs were also upregulated, and 7 of the drought-downregulated TFs were also downregulated in abs under normal conditions. Seventeen TFs did not show obvious changes in expression in SIBR/1OE plants compared with MM plants; 4 TFs, ERF (Solyc06g068360.3), AP2-ERF (Solyc10g084340.2), MYB75 (Solyc10g086250.2) and bHLH079 (Solyc02g078130.3), had the same expression direction, while 4 TFs, WRKY33 (Solyc09g014990.4), WRKY46 (Solyc08g067340.4), MYB76 (Solyc05g008250.2) and bHLH150 (Solyc09g065100.3), had opposite expression changes in SIBRI1OE plants and drought-exposed plants (Fig. 8B; Table S5). Eighteen of 25 TFs had the same expression direction in absDS compared with MMDS, and 7 of 25 TFs were not obviously changed (Fig. 9B; Table S7). In abs plants, reduced BR signalling may upregulate or downregulate drought-related TFs, which further regulate drought stressrelated gene expression and improve drought resistance under both normal conditions and drought conditions.

\section{Validation of RNA-Seq data by qRT-PCR analysis}

To validate the RNA-seq data, we selected 15 DEGs of MM vs abs for qRT-PCR determination. We further compared the results obtained from qRT-PCR with those generated from the RNA-seq data. The trends of expression were consistent for all transcripts in both analyses, with a correlation coefficient of $R^{2}=0.883$ (Fig. 10). These results confirmed the reliability of the RNA-seq data.

\section{Discussion}

Plants frequently encounter various environmental stresses, such as drought, salt and extreme temperature, which severely affect plant growth and yield. In many previous studies, it has been reported that the application of BRs can improve the drought resistance of plants. In recent years, we found that $S / B R / 1$-overexpressing plants from the tomato cultivar Micro-Tom have increased BR signaling but decreased drought resistance. We further found that SIBR/1 weak mutants in the tomato cultivar Money Maker (MM) background (altered brassinolide sensitivity, abs) exhibited delayed growth and enhanced drought resistance[31]. To further understand the relationship between the S/BR/1 expression level and drought resistance in tomato, we obtained S/BR/1 overexpressing and S/BR/1 weak mutant plants in the same background to examine their differences in drought resistance. The expression levels of $S / B R / 1$ in transgenic plants were significantly higher than those in MM plants (Figs. 1A, B). Furthermore, the transgenic plants already exhibited SIBRI1 protein expression. In addition, the expression of the BR biosynthetic genes DWARF and CPD was significantly inhibited in the transgenic plants (Figs. 1C, D). All results indicated that the $S / B R / 1$ transgenic plants had obviously increased BR signaling. 
Abiotic stress can lead to the reduction of photosynthesis through stomata-dependent and stomataindependent pathways. If $\mathrm{Ci}$ and $\mathrm{Gs}$ are reduced simultaneously, the reduction of $\mathrm{Pn}$ can be considered to be primarily due to stomatal factors. In contrast, if $\mathrm{Gs}$ is reduced but $\mathrm{Ci}$ does not change or is increased, the reduction in Pn can be considered to be due to nonstomatal factors [37-40]. In our study, drought stress decreased the Pn of all plants. Furthermore, the Pn and Tr of abs plants were significantly higher than those of other plants, while the $\mathrm{Ci}$ of abs plants was significantly lower than that of other plants under drought conditions (Figs. 3A-D). These results were consistent with BRI1 RNAi plants having higher photosynthetic capacity than wild-type plants in Brachypodium distachyon under drought stress[29]. In contrast, the $\mathrm{Pn}$ and $\mathrm{Tr}$ of SIBR/1-overexpressing plants were significantly lower than those of other plants, while the $\mathrm{Ci}$ was significantly higher than that of other plants under drought conditions (Figs. 3A, C, D). Therefore, the photosynthetic capacity of abs plants was less affected by drought stress. These results indicated that the reduction in $\mathrm{Pn}$ in all plants could be attributed to stomatal factors and nonstomatal factors. Nonstomatal factors may include the reduced activity and efficiency of partial enzymes of the Calvin cycle, destroyed photosynthetic apparatus and other organelles and slowed transport of photosynthetic products [41].

Normally, the formation and elimination of ROS are balanced and steady, but this balance is destroyed when plants are subjected to drought stress. In this study, drought stress obviously increased the accumulation of $\mathrm{H}_{2} \mathrm{O}_{2}$ and $\mathrm{O}_{2}{ }^{-}$in all plants, as determined by NBT and DAB staining (Fig. 4A, B). The accumulation of $\mathrm{H}_{2} \mathrm{O}_{2}$ and $\mathrm{O}_{2}{ }^{-}$in abs leaves was lower than that in MM leaves, but S/BR/1-

overexpressing leaves accumulated much more $\mathrm{H}_{2} \mathrm{O}_{2}$ and $\mathrm{O}_{2}{ }^{-}$than $\mathrm{MM}$ leaves (Fig. 4A, B). Therefore, abs plants had lower ROS levels, and SIBR/1 overexpressing plants had higher ROS levels, than MM plants under drought stress. These results were consistent with the observation that the SOD, POD, CAT and APX activities of abs plants were significantly higher than those of other plants under drought stress (Fig. 4CF). The activities of these enzymes in SIBR/7-overexpressing plants were significantly lower than those in $\mathrm{MM}$ and abs plants after drought stress. Furthermore, our RNA-seq data indicated that 11 oxidoreductase activity genes were induced in abs plants under normal conditions. Seven of 11 genes were upregulated in MM plants under drought stress. However, most of these genes were not obviously changed in SIBRI1OE plants. In addition, the expression of 9 of these same 11 genes was higher in absDS plants than in MMDS plants (Fig. 9A). These results indicated that abs plants may decrease reactive oxygen content by inducing oxidoreductase activity gene expression and further enhance antioxidant enzyme activities under drought conditions.

The pleiotropic roles of BR are complex, including developmental processes and multiple types of stress tolerance in plants. To further understand the relationship between the SIBR/1 expression level and drought resistance in tomato, we used RNA-Seq to characterize the influence of the S/BR/1 expression level on gene expression under normal and drought conditions. We focused on comparing the differences in gene expression between MM and MMDS, MM and $a b s, \mathrm{MM}$ and SIBR/1OE and MMDS and absDS. We found that $768(53.9 \%)$ of 1425 drought-induced and 418 (49.8\%) of 840 drought-repressed genes were regulated by abs in the same direction under normal conditions (Fig. 6A, B). There were 158 drought- 
induced and 43 drought-repressed genes that were further upregulated and downregulated in absDS, respectively (Fig. 6A, B). This result was consistent with the observation that RNAi of a BRI1 homologue in Brachypodium distachyon decreased the growth potential of plants while increasing their tolerance to drought and drought-responsive gene expression[29]. ABA plays a crucial role in drought resistance. Drought stress usually induces the expression of $A B A$ biosynthesis genes, which further increases the ABA content and eventually enhances the drought resistance of plants[31]. Therefore, we compared stress-related metabolic pathways identified in GO enrichment analyses. Our results indicated that drought stress upregulated 4 ABA biosynthesis genes that were already induced in abs plants under normal conditions. Additionally, the expression of 2 ABA biosynthesis genes was higher in absDS plants than in MMDS plants (Table S4). These results indicated that abs may increase ABA contents and further improve drought resistance by inducing $A B A$ biosynthetic gene expression under normal and drought conditions.

Dopamine also plays an important role in the drought resistance of plants[42]. A previous study showed that dopamine could regulate photosynthetic oxygen reduction and the photophosphorylation of chloroplasts[43]. Furthermore, dopamine could increase the drought resistance of apple seedlings by improving the photosynthetic capacity and antioxidant enzyme activity of the plants[44]. Our results indicated that 13 polyamine biosynthetic genes were upregulated in abs plants under normal conditions. Eleven of these 13 were induced in MM under drought stress. Furthermore, the expression of 8 genes was higher in absDS plants than in MMDS plants. However, most of these genes did not show obvious changes in expression in SIBRI1OE plants compared with MM plants (Table S5). Therefore, these results indicated that abs may increase polyamine contents and further improve drought resistance by inducing polyamine biosynthetic gene expression under normal and drought conditions.

TFs play an important role in drought stress signalling. Some TFs are major components in signalling networks, including WRKY, NAC, ERF, bHLH, bZIP and MYB. In our study, 25 transcription factor-encoding genes were differentially expressed between different treatments, mainly from the WRKY, ERF, bHLH and MYB families. Furthermore, the most abundant TF members were from the WRKY transcription factor family (Fig. 9B, C; Table S7). Previous studies found that WRKY38 participates in the drought stress response and that WRKY4O is involved in ABA signalling [45, 46]. The overexpression of OsWRKY3O obviously improved drought tolerance in rice. Compared to the MM group, the MMDS group showed upregulation of drought-related TFs such as WRKY81, WRKY33 and WRKY46. However, in abs plants, the expression of these genes was already upregulated under normal conditions. Moreover, the expression of these genes in absDS plants was higher than that in MMDS plants, while the expression of these genes was downregulated in SIBRI1OE plants compared with MM plants (Fig. 9B; Table S7). Therefore, droughtrelated WRKY TFs may be upregulated in abs plants and further improve drought resistance under both normal conditions and drought conditions.

AP2/ERF TFs are involved in the regulation of plant drought responses and plant growth (Phukan et al., 2017; Xie et al., 2019). The overexpression of stress-inducible AP2/ERF TFs inhibited plant growth but improved the drought resistance of transgenic plants. TINY positively regulates drought responses by 
inducing drought-responsive genes and promoting abscisic acid-regulated stomatal closure. BR signalling negatively regulates TINY through BIN2 phosphorylation, and TINY inhibits BR-mediated growth through antagonistic TINY-BES1 interactions. Three AP2/ERF TFs each were upregulated and downregulated in the MMDS group compared with the MM group. However, these same genes were already upregulated and downregulated under normal conditions in abs plants (Fig. 9B). However, the expression of most of the same genes was not different in S/BR/1OE plants compared to MM plants. We found similar results for some bHLH and MYB family TFs in abs plants and S/BR/1OE plants (Fig. 9B). Therefore, in abs plants, bri1 mutation leads to reduced BR signalling and may upregulate or downregulate drought-related TFs, which further regulate drought stress-related gene expression and ultimately improve drought resistance both under normal conditions and drought conditions. These results were consistent with the growth phenotype of abs.

\section{Conclusions}

In conclusion, physiological analyses revealed that abs plants were more drought resistant than MM plants through two mechanisms. First, abs plants had a higher net photosynthetic rate and relative water content as well as lower wilting and electrolyte leakage; second, abs plants had lower $\mathrm{H}_{2} \mathrm{O}_{2}$ and $\mathrm{O}_{2}{ }^{-}$ accumulation through higher antioxidant enzyme activities under drought conditions. RNA-Seq was used to characterize the influence of $S / B R / 1$ expression level on drought tolerance in tomato. In total, 768 (53.9\%) of 1425 drought-induced genes and 418 (49.8\%) of 840 drought-repressed genes were regulated in abs in the same direction under normal conditions. Moreover, 158 drought-induced and 43 droughtrepressed genes were further upregulated and downregulated in abs plants under drought conditions, respectively. We further found that abs regulated the expression of genes related to the metabolism of ABA and polyamine biosynthesis, as well as oxidoreductase activity genes under normal and drought conditions, by GO enrichment analyses. Furthermore, abs may affect tomato drought tolerance by regulating the expression of WRKY, ERF, bHLH and MYB transcription factors. Therefore, our study provides valuable information for future breeding to reduce the expression of $S / B R / 1$ and improve drought resistance without affecting plant growth.

\section{Methods}

\section{Plant materials, growth conditions and plant transformation}

Seeds of tomato cultivar Money Maker (MM), abs (S/BR/1 weak mutant) and T2-generation transgenic tomato plants were germinated at $28^{\circ} \mathrm{C}$ in Petri plates lined with two layers of filter paper moistened with deionized water. Tomato MM and abs seeds were obtained from Northwest A\&F University Xiaofeng Wang laboratory. The germinated seeds were then sown in plastic pots $(8 \mathrm{~cm} \times 8 \mathrm{~cm} \times 9 \mathrm{~cm})$ filled with $70 \mathrm{~g}$ of a mixture of peat and vermiculite $(\mathrm{v} / \mathrm{v}=7: 3)$ at one seed per pot. Seedlings were grown in a controlled environment room with a temperature of $25^{\circ} \mathrm{C}$, photosynthetic photon flux density (PPFD) of $500 \mu \mathrm{mol} \mathrm{m} \mathrm{m}^{-2} \mathrm{~s}^{-1}$, and a photoperiod of $16 / 8 \mathrm{~h}$ (day/night). 
The overexpression of 35S:SIBR/1 transgenic tomato was obtained using the method of Nie et al. [33, 34]. Two independent homozygous SIBR/1 overexpression plants (SIBR/1-OE-6 and SIBR/1-OE-7) were used for drought experiments because of high expression levels of SIBR/1. abs were sown $30 \mathrm{~d}$ earlier than other tomato plants because abs plants slowly grow.

\section{Drought experiments}

abs seeds were sown $30 \mathrm{~d}$ earlier than other tomato seeds because abs plants slowly grow. All plants were grown to the six-leaf stage. Plants of the same size were selected for subsequent experiments. The selected plants were unwatered for 12 days. There were 40 seedlings in each group.

\section{Measurements of relative water content (RWC) and electrolyte leakage}

Fresh fully expanded leaves were harvested for measuring the RWC and electrolyte leakage. RWC and electrolyte leakage were measured according to Liu et al. [35].

\section{Measurement of photosynthetic parameters}

The net photosynthetic rate ( $\mathrm{Pn})$, stomatal conductance (Gs), intercellular $\mathrm{CO}_{2}$ concentration ( $\mathrm{Ci}$ ), and transpiration rate $(\mathrm{Tr})$ of the seedlings were measured by using a portable photosynthesis system (LI6400-40, Li-Cor, Lincoln, NE, USA). All measurements were carried out at $400 \mu \mathrm{mol} \mathrm{mol}^{-1} \mathrm{CO}_{2}, 25^{\circ} \mathrm{C}$, and $500 \mu \mathrm{mol} \mathrm{m} \mathrm{m}^{-2} \mathrm{~s}^{-1}$ light intensity. For each measurement, 8 leaves were measured from different seedlings per treatment.

\section{Antioxidant enzyme activities and the accumulation of ROS}

Antioxidant enzyme activities and the accumulation of ROS were performed in accordance with the method of Nie et al.[31].

\section{Western blot analysis}

Total proteins were extracted from the young leaves $(0.2 \mathrm{~g})$ of 25 -day-old different tomato plants. The procedure was performed as previously described [36].

\section{RNA extraction and Quantitative real-time PCR (qRT-PCR) analysis}

Total RNA was extracted and reverse-transcribed according to Nie et al. [33]. qRT-PCR was performed following the three-step protocol of BioEasy Master Mix (Bioer Technology) in a CFX96 real-time system (Bio-Rad) as described in the manufacturer's instructions. Each assay consisted of three biological replicates. We used UBI3 gene as the internal control. All specific primer sequences are listed in Table S1.

\section{RNA-Seq and transcriptomic analysis}

Total RNA samples were collected from the meristem and young leaves by using the RNAprep Pure Plant Kit (Tiangen Biotech) following the manufacturer's instructions. RNA quality and concentration were assessed by using an Agilent 2100 Bioanalyzer (Agilent Technologies, USA) and NanoDrop 2000 spectrophotometer (Thermo Fisher Scientific, USA). For the transcriptome analyses, RNA-seq libraries were prepared from cDNA by Majorbio and sequenced on an Illumina HiSeq 4000. Raw sequences were quality-filtered by SeqPrep (https://github.com/jstjohn/SeqPrep) and Sickle 
(https://github.com/najoshi/sickle) and mapped to the Solanum_lycopersicum SL4.0 reference genome (https://www.solgenomics.net/organism/Solanumlycopersicum/genome/) by using TopHat2 (http://ccb.jhu.edu/software/tophat/index.shtml). DESeq2 was used for the differential expression analysis of RNA-Seq expression profiles. Absolute values of log2 (fold change) $>=1$ and p-adjusted $<0.05$ were set as thresholds. The genes that were expressed at significantly different levels were subjected to KEGG, GO function analysis and GO enrichment analysis. The metabolic pathway heatmap was generated using Multiple Array Viewer.

The RNA-Seq samples were divided into six groups: (1) Money Maker normal sample before drought stress (MM); (2) abs normal sample before drought stress (abs); (3) SIBR/1-OE-7 normal sample before drought stress (SIBR/1OE); (4) Money Maker drought-stressed $8 \mathrm{~d}$ sample (MMDS); (5) abs droughtstressed $8 \mathrm{~d}$ sample (absDS); and (6) SIBR/1-OE-7 drought-stressed $8 \mathrm{~d}$ sample (S/BR/1OEDS).

\section{Statistical Analysis}

All data in this study were analyzed using SPSS version 17.0 and the LSD test. The means and standard errors were calculated, and $\mathrm{P}<0.05$ was considered statistically significant in comparisons with $\mathrm{MM}$ plants.

\section{Abbreviations}

BRs: Brassinosteroids; ABA, Abscisic acid; abs: altered brassinolide sensitivity; MM: Money Maker; 35S:Constitutive cauliflower mosaic virus 35S promoter; BRI1:BRASSINOSTEROID-INSENSITIVE 1; ROS: reactive oxygen species; BAK1: BRI1-ASSOCIATED RECEPTOR KINASE1; BES1: BRI1-EMS SUPPRESSOR1; BZR1: BRASSINAZOLERESISTANT1; CBB: Coomassie brilliant blue; CPD: CONSTITUTIVE PHOTOMORPHOGENESIS AND DWARF; DWARF: 6-DEOXOCASTASTERONE OXIDASE; BIN2:

brassinosteroid insensitive 2; RD26: RESPONSIVE TO DESICCATION26; TaBZR2: Wheat brassinazoleresistant 2; TaGST1: T. aestivum glutathione-transferase-1; RWC: relative water content; Pn: net photosynthetic rate; Gs: stomatal conductance; $\mathrm{Tr}$ : transpiration rate; $\mathrm{Ci}$ : intercellular $\mathrm{CO}_{2}$ concentration; $\mathrm{H}_{2} \mathrm{O}_{2}$ : hydrogen peroxide; $\mathrm{O}_{2}^{-}$: superoxide; SOD, Superoxide dismutase; CAT: Catalase; POD: Peroxidase; APX: Ascorbate peroxidase; RNA-seq: RNA sequencing;

\section{Declarations}

\section{Ethics approval and consent to participate}

Not applicable.

\section{Consent for publication}

Not Applicable.

\section{Availability of data and materials}


All data generated or analyzed during this study are included in this published article and its supplementary information files. The datasets and plant materials used during the current study are available from the corresponding author on reasonable request.

\section{Competing interests}

The authors declare that they have no competing interests.

\section{Funding}

This research was financially supported by the Science and Technology Department of Sichuan Province (Nos. 467630) and the Fundamental Research Funds of China West Normal University (Nos. 412836). The supporter provided financial help for experiment design, data collection, data analysis and manuscript writing.

\section{Author contributions}

WD and NS planned and designed the research. WD and NS performed the experiments. WD, YZ and NS analyzed the data. WD and NS wrote the manuscript. All authors read and approved the final manuscript.

\section{Acknowledgements}

We thank the Northwest A\&F University Xiaofeng Wang laboratory for giving us Tomato MM and abs seeds.

\section{Authors' Information}

Key Laboratory of Southwest China Wildlife Resources Conservation (Ministry of Education), College of Life Science, China West Normal University, Nanchong 637009, Sichuan, China

Shu-Ming Nie, Zai-Jun Yang \& Dan Wang

\section{References}

1. Yordanov I, Velikova V, Tsonev T: Plant responses to drought, acclimation, and stress tolerance. Photosynthetica 2000, 38(2):171-186.

2. Jaleel CA, Manivannan P, Lakshmanan GMA, Gomathinayagam M, Panneerselvam R: Alterations in morphological parameters and photosynthetic pigment responses of Catharanthus roseus under soil water deficits. Colloid Surface B 2008, 61(2):298-303.

3. Talbi S, Romero-Puertas MC, Hernandez A, Terron L, Ferchichi A, Sandalio LM: Drought tolerance in a Saharian plant Oudneya africana: Role of antioxidant defences. Environ Exp Bot 2015, 111:114-126.

4. Chaves MM, Oliveira MM: Mechanisms underlying plant resilience to water deficits: prospects for water-saving agriculture. J Exp Bot 2004, 55(407):2365-2384. 
5. Morales CG, Pino MT, del Pozo A: Phenological and physiological responses to drought stress and subsequent rehydration cycles in two raspberry cultivars. Sci Hortic-Amsterdam 2013, 162:234-241.

6. Sofo A, Tuzio AC, Dichio B, Xiloyannis C: Influence of water deficit and rewatering on the components of the ascorbate-glutathione cycle in four interspecific Prunus hybrids. Plant Sci 2005, 169(2):403412.

7. Liu B, Li M, Cheng L, Liang D, Zou Y, Ma F: Influence of rootstock on antioxidant system in leaves and roots of young apple trees in response to drought stress. Plant Growth Regulation 2012, 67(3):247256.

8. Boaretto LF, Carvalho G, Borgo L, Creste S, Landell MG, Mazzafera P, Azevedo RA: Water stress reveals differential antioxidant responses of tolerant and non-tolerant sugarcane genotypes. Plant physiology and biochemistry : PPB / Societe francaise de physiologie vegetale 2014, 74:165-175.

9. Wang S, Liang D, Li C, Hao Y, Ma F, Shu H: Influence of drought stress on the cellular ultrastructure and antioxidant system in leaves of drought-tolerant and drought-sensitive apple rootstocks. Plant physiology and biochemistry : PPB / Societe francaise de physiologie vegetale 2012, 51:81-89.

10. Clouse SD, Sasse JM: Brassinosteroids: Essential regulators of plant growth and development. Annu Rev Plant Phys 1998, 49:427-451.

11. Kim TW, Wang ZY: Brassinosteroid Signal Transduction from Receptor Kinases to Transcription Factors. Annu Rev Plant Biol 2010, 61:681-704.

12. Vert G, Nemhauser JL, Geldner N, Hong FX, Chory J: Molecular mechanisms of steroid hormone signaling in plants. Annu Rev Cell Dev Bi 2005, 21:177-201.

13. Wang XF, Kota U, He K, Blackburn K, Li J, Goshe MB, Huber SC, Clouse SD: Sequential transphosphorylation of the BRI1/BAK1 receptor kinase complex impacts early events in brassinosteroid signaling. Dev Cell 2008, 15(2):220-235.

14. Yu JQ, Huang LF, Hu WH, Zhou YH, Mao WH, Ye SF, Nogues S: A role for brassinosteroids in the regulation of photosynthesis in Cucumis sativus. J Exp Bot 2004, 55(399):1135-1143.

15. Li JM, Nagpal P, Vitart V, McMorris TC, Chory J: A role for brassinosteroids in light-dependent development of Arabidopsis. Science 1996, 272(5260):398-401.

16. Wenhai H, Lifeng H, Weihua M, Yanhong Z, Yu A: Role of Brassinosteroids in the Regulation of Photosynthetic Apparatus in Cucumber Leaves. Acta Horticulturae Sinica 2006, 33(4):762-766.

17. Wu G, Wang X, Li X, Kamiya Y, Otegui MS, Chory J: Methylation of a phosphatase specifies dephosphorylation and degradation of activated brassinosteroid receptors. Science Signaling 2011, 4(172):ra29.

18. Qiao S, Sun S, Wang L, Wu Z, Li C, Li X, Wang T, Leng L, Tian W, Lu T: The RLA1/SMOS1 Transcription Factor Functions with OsBZR1 to Regulate Brassinosteroid Signaling and Rice Architecture. Plant Cell 2017, 29(2):292.

19. Wang ZY, Seto H, ., Fujioka S, ., Yoshida S, ., Chory J, . BRI1 is a critical component of a plasmamembrane receptor for plant steroids. Nature 2001, 410(6826):380-383. 
20. Li J, Chory J: A putative leucine-rich repeat receptor kinase involved in brassinosteroid signal transduction. Cell 1997, 90(5):929-938.

21. Qinsong, Thomas, Vain, Corrado, Viotti, Siamsa, Doyle, Danuse, Tarkowska, Ondrej: Vacuole Integrity Maintained by DUF300 Proteins Is Required for Brassinosteroid Signaling Regulation. Molecular Plant 2018, 11(4):553-567.

22. Wang X, Kota U, He K, Blackburn K, Li J, Goshe MB, Huber SC, Clouse SD: Sequential transphosphorylation of the BRI1/BAK1 receptor kinase complex impacts early events in brassinosteroid signaling. Dev Cell 2008, 15(2):220-235.

23. T-DNA tagged knockout mutation of rice OsGSK1, an orthologue of Arabidopsis BIN2, with enhanced tolerance to various abiotic stresses. Plant Molecular Biology 2007, 65(4):453-466.

24. Chung Y, Choe V, Fujioka S, Takatsuto S, Han M, Jeon JS, Park YI, Lee KO, Choe S: Constitutive activation of brassinosteroid signaling in the Arabidopsis elongated-D/bak1 mutant. Plant Molecular Biology 2012, 80(4-5):489-501.

25. Ye H, Liu S, Tang B, Chen J, Xie Z, Nolan TM, Jiang H, Guo H, Lin HY, Li L: RD26 mediates crosstalk between drought and brassinosteroid signalling pathways. Nature Communications 2017, 8:14573.

26. Chen J, Nolan T, Ye H, Zhang M, Tong H, Xin P, Chu J, Chu C, Li Z, Yin Y: Arabidopsis WRKY46, WRKY54, and WRKY70 Transcription Factors Are Involved in Brassinosteroid-Regulated Plant Growth and Drought Responses. The Plant Cell 2017:tpc.00364.02017.

27. Xie Z, Nolan T, Jiang H, Tang B, Yin Y: The AP2/ERF Transcription Factor TINY Modulates Brassinosteroid-Regulated Plant Growth and Drought Responses in Arabidopsis. The Plant Cell 2019, 31(8):tpc.00918.02018.

28. Xiao-Yu, Cui, Yuan, Gao, Jun, Guo, Tai-Fei, Wei-Jun, Zheng, Yong-Wei: BES/BZR Transcription Factor TaBZR2 Positively Regulates Drought Responses by Activation of TaGST1. Plant physiology 2019.

29. Feng Y, Yin Y, Fei S: Down-regulation of BdBRI1, a putative brassinosteroid receptor gene produces a dwarf phenotype with enhanced drought tolerance in Brachypodium distachyon. Plant ence 2015, 234:163-173.

30. Overexpression of the vascular brassinosteroid receptor BRL3 confers drought resistance without penalizing plant growth. Nature communications 2018.

31. Nie S, Huang S, Wang S, Mao Y, Liu J, Ma R, Wang X: Enhanced brassinosteroid signaling intensity via SIBRI1 overexpression negatively regulates drought resistance in a manner opposite of that via exogenous BR application in tomato. Plant Physiology \& Biochemistry 2019.

32. Bajwa VS, Wang X, Blackburn RK, Goshe MB, Mitra SK, Williams EL, Bishop GJ, Krasnyanski S, Allen G, Clouse HSD: Identification and Functional Analysis of Tomato BRI1 and BAK1 Receptor Kinase Phosphorylation Sites. Plant Physiology 2013, 163(1):30-42.

33. Nie S, Huang S, Wang S, Cheng D, Liu J, Lv S, Li Q, Wang X: Enhancing Brassinosteroid Signaling via Overexpression of Tomato (Solanum lycopersicum) SIBRI1 Improves Major Agronomic Traits. Frontiers in plant science 2017, 8:1386. 
34. Park SH, Morris JL, Park JE, Hirschi KD, Smith RH: Efficient and genotype-independent Agrobacterium - Mediated tomato transformation. Journal of Plant Physiology 2003, 160(10):1253-1257.

35. Liu H, Yu C, Li H, Ouyang B, Wang T, Zhang J, Wang X, Ye Z: Overexpression of ShDHN, a dehydrin gene from Solanum habrochaites enhances tolerance to multiple abiotic stresses in tomato. Plant Sci 2015, 231:198-211.

36. Wang Q, Wang S, Gan S, Wang X, Liu J, Wang X: Role of Specific Phosphorylation Sites of Arabidopsis Brassinosteroid-Insensitive 1 Receptor Kinase in Plant Growth and Development. Journal of Plant Growth Regulation 2016, 35(3):1-15.

37. Yin $\mathrm{CY}$, Peng YH, Zang RG, Zhu YP, Li CY: Adaptive responses of Populus kangdingensis to drought stress. Physiol Plantarum 2005, 123(4):445-451.

38. Jia H, Oguchi R, Hope AB, Barber J, Chow WS: Differential effects of severe water stress on linear and cyclic electron fluxes through Photosystem I in spinach leaf discs in $\mathrm{CO}(2)$-enriched air. Planta 2008, 228(5):803-812.

39. Begcy K, Mariano ED, Gentile A, Lembke CG, Zingaretti SM, Souza GM, Menossi M: A Novel StressInduced Sugarcane Gene Confers Tolerance to Drought, Salt and Oxidative Stress in Transgenic Tobacco Plants. Plos One 2012, 7(9).

40. Zhang LD, Zhang LX, Sun JL, Zhang ZX, Ren HZ, Sui XL: Rubisco gene expression and photosynthetic characteristics of cucumber seedlings in response to water deficit. Sci HorticAmsterdam 2013, 161:81-87.

41. Sapeta H, Costa JM, Lourenco T, Maroco J, van der Linde P, Oliveira MM: Drought stress response in Jatropha curcas: Growth and physiology. Environ Exp Bot 2013, 85:76-84.

42. Liang B, Gao T, Qi Z, Ma C, Qi C, Wei Z, Li C, Chao L, Ma F: Effects of Exogenous Dopamine on the Uptake, Transport, and Resorption of Apple lonome Under Moderate Drought. Frontiers in plant science 2018, 9:755.

43. Elstner EF, Konze JRR, Stoffer SC: Ethylene Formation in Sugar Beet Leaves. Plant Physiology 1976, 58(2):163-168.

44. Li P, Yang H, Wang L, Liu H, Huo H, Zhang C, Liu A, Zhu A, Hu J, Lin Y: Physiological and Transcriptome Analyses Reveal Short-Term Responses and Formation of Memory Under Drought Stress in Rice. Frontiers in Genetics 2019, 10.

45. Han C, Lai Z, Shi J, Yong X, Chen Z, Xu X: Roles of arabidopsis WRKY18, WRKY40 and WRKY60 transcription factors in plant responses to abscisic acid and abiotic stress. Bmc Plant Biology 2010, 10(1):281.

46. Albayrak G, Yoruk E, Diken O: Quantitative gene expression analysis of WRKY38 and DREB2 transcription factors responsible for drought and salt tolerance in barley (Hordeum vulgare L.). New Biotechnology 2012, 29(Suppl):S22-S22.

\section{Figures}


A

IB: anti-flag

MM SIBR/1-OE-6 SIBR/1-OE-7

CBB

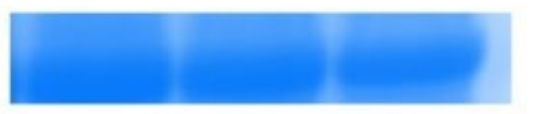

BRI1-Flag

B
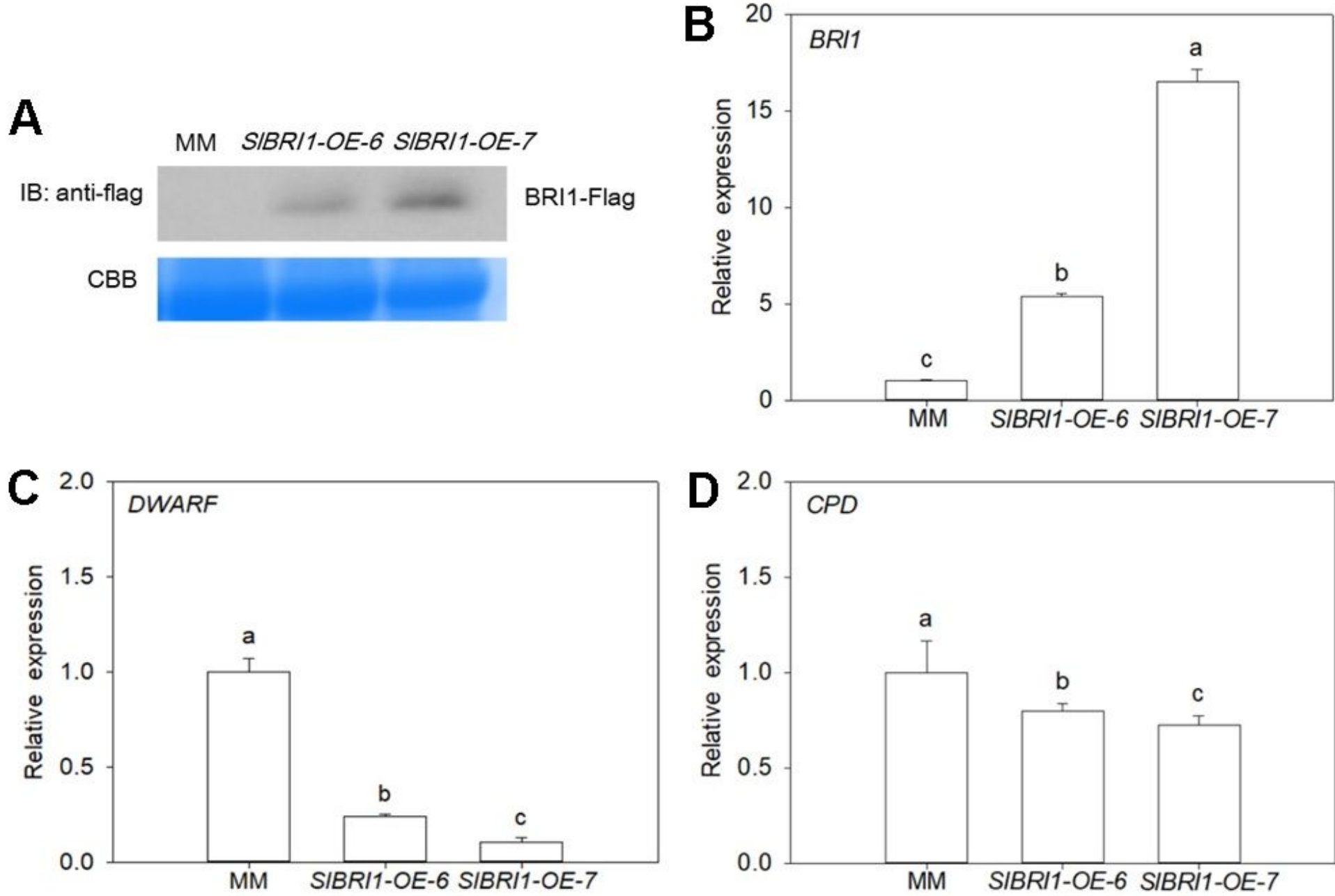

\section{Figure 1}

SIBRI1, DWARF and CPD expression in Money Maker (MM) and SIBRI1-overexpressing plants. (A) Transgenic BRI1 protein levels examined by immunoblotting with anti-Flag antibodies. CBB, Coomassie brilliant blue. The Western blot has been cropped and uncropped blot have be included in the additional files. (B) The relative transcript levels of SIBRI1. (C) The relative transcript levels of DWARF. (D) The relative transcript levels of $C P D$. Data values are the means $\pm S D$ of three independent biological samples. Different letters indicate significant differences according to Tukey's test $(P<0.05)$. The different samples were derived from the same experiment. 

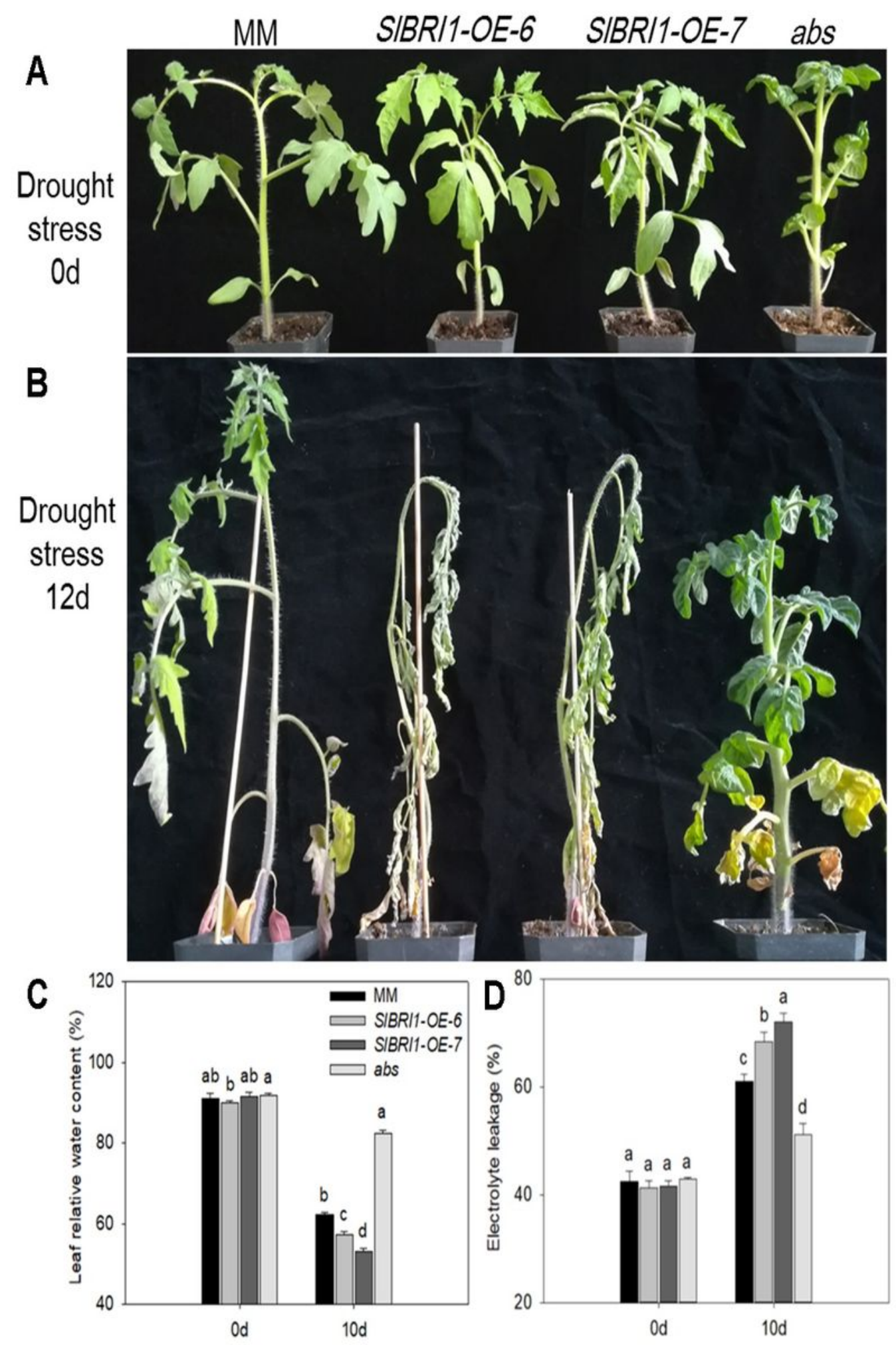

Figure 2

MM, SIBRI1 overexpression and abs plants under drought stress. (A) and (B) Phenotypes of MM, SIBRI1OE-6, SIBRI1-OE-7, and abs plants after 0 and $12 \mathrm{~d}$ of drought stress, respectively. (C) Leaf relative water content (RWC) of WT, SIBRI1-OE-6, and SIBRI1-OE-7 plants after 0 and $10 \mathrm{~d}$ of drought stress. (D) Electrolyte leakage. Data values are the means \pm SD of three independent biological samples. Different letters indicate significant differences according to Tukey's test $(P<0.05)$. 

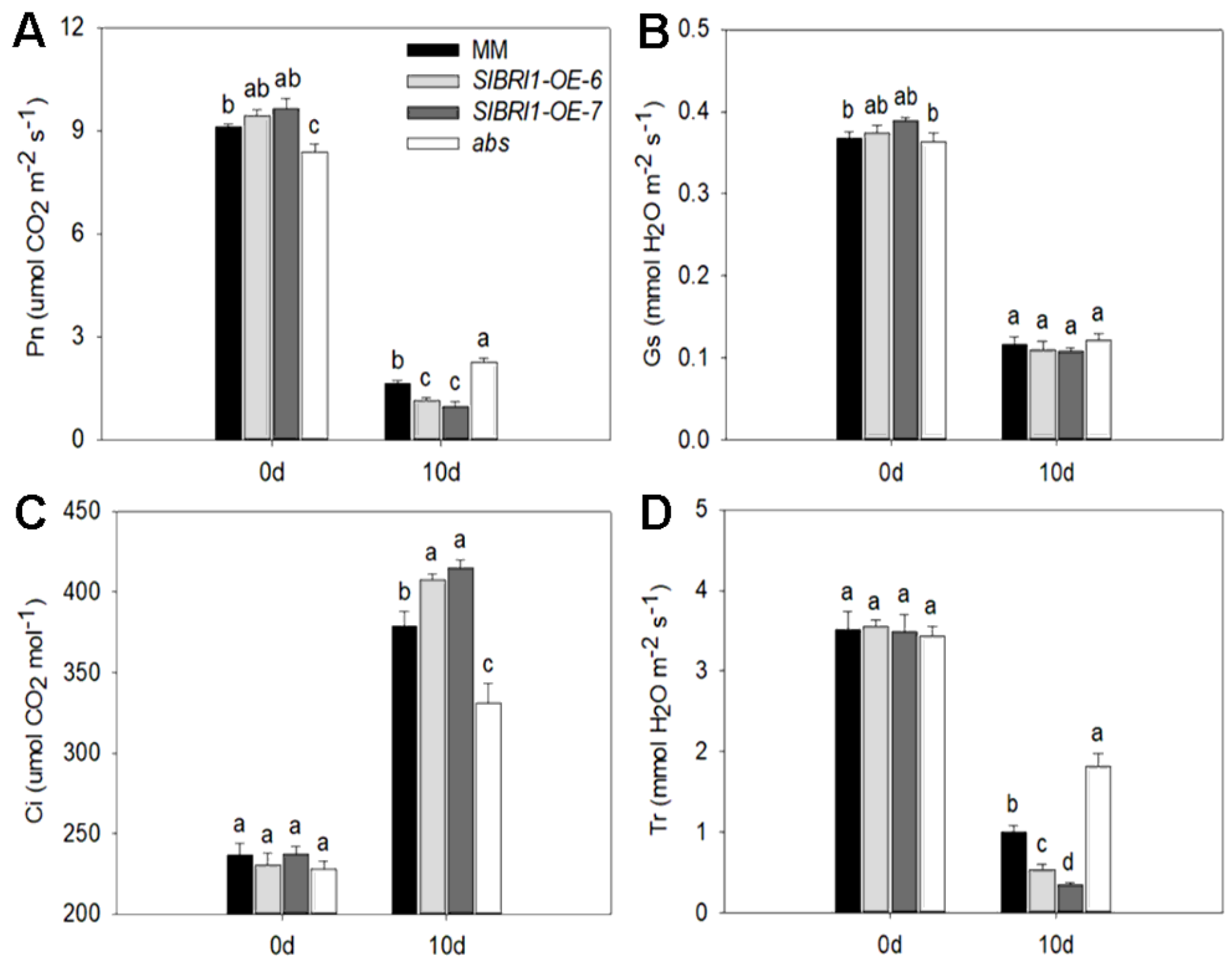

Figure 3

Effects of different SIBRI1 expression levels on gas exchange parameters in tomato seedlings under drought stress. (A) Photosynthetic rate (Pn), (B) Stomatal conductance (Gs), (C) Intercellular CO2 concentration $(\mathrm{Ci})$, and $(\mathrm{D})$ Transpiration rate. Data values are the means \pm SD of three independent biological samples. Different letters indicate significant differences according to Tukey's test $(P<0.05)$.v 


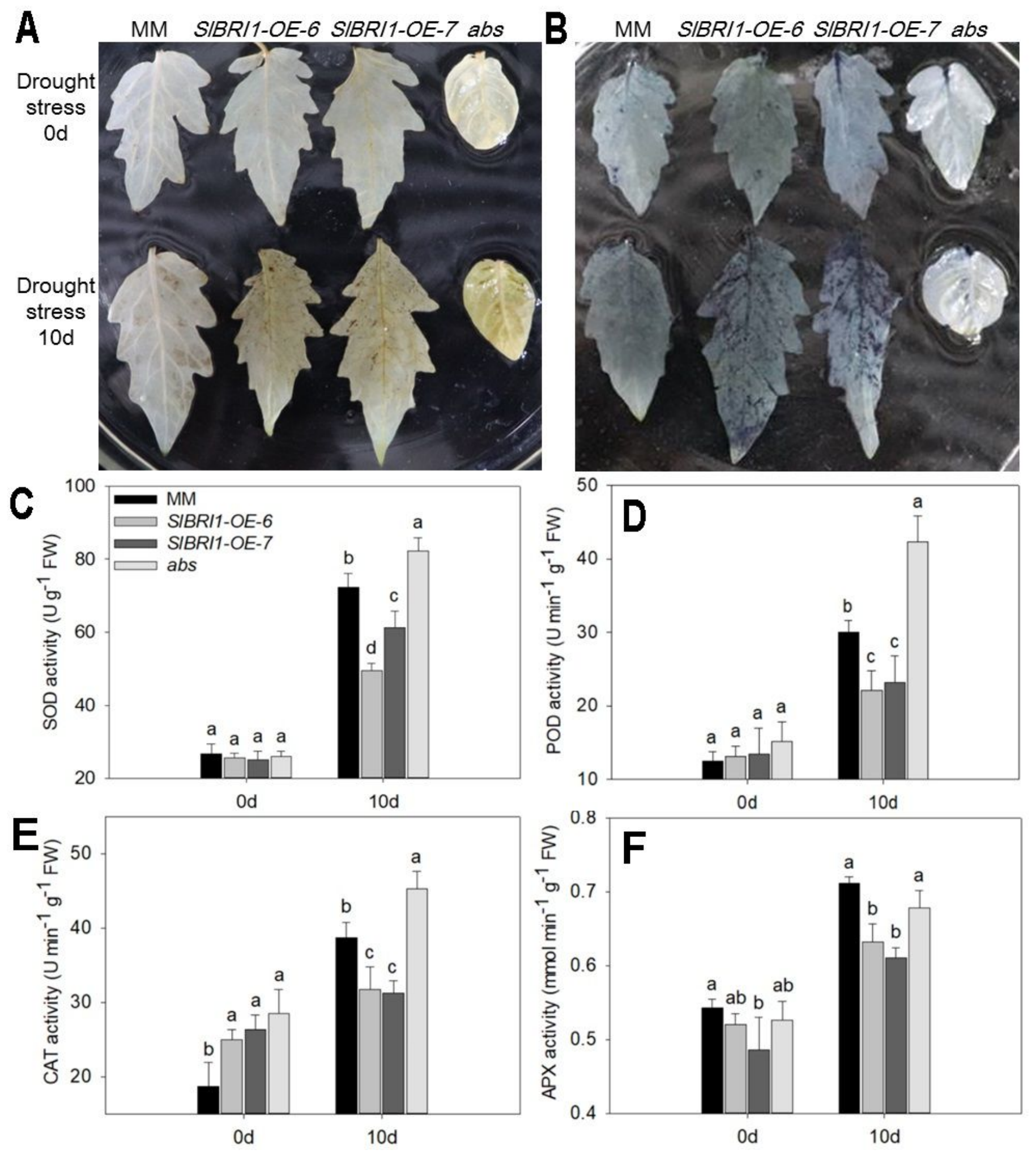

Figure 4

Effects of different SIBRI1 expression levels on reactive oxygen species (ROS) accumulation and antioxidant enzyme activity in tomato seedlings under drought stress. (A) The accumulation of hydrogen peroxide (H2O2) in MM, SIBRI1-OE-6, SIBRI1-OE-7, and abs plants after 0 and $10 \mathrm{~d}$ of drought stress. (B) The accumulation of superoxide (O2-). (C) Superoxide dismutase (SOD) activity in MM, SIBRI1-OE-6, SIBRI1-OE-7, and abs plants after 0 and 10 days of drought stress. (D) Peroxidase (POD) activity. (E) 
Catalase (CAT) activity. (F) Ascorbate peroxidase (APX) activity. Data values are the means \pm SD of three independent biological samples. Different letters indicate significant differences according to Tukey's test $(P<0.05)$.

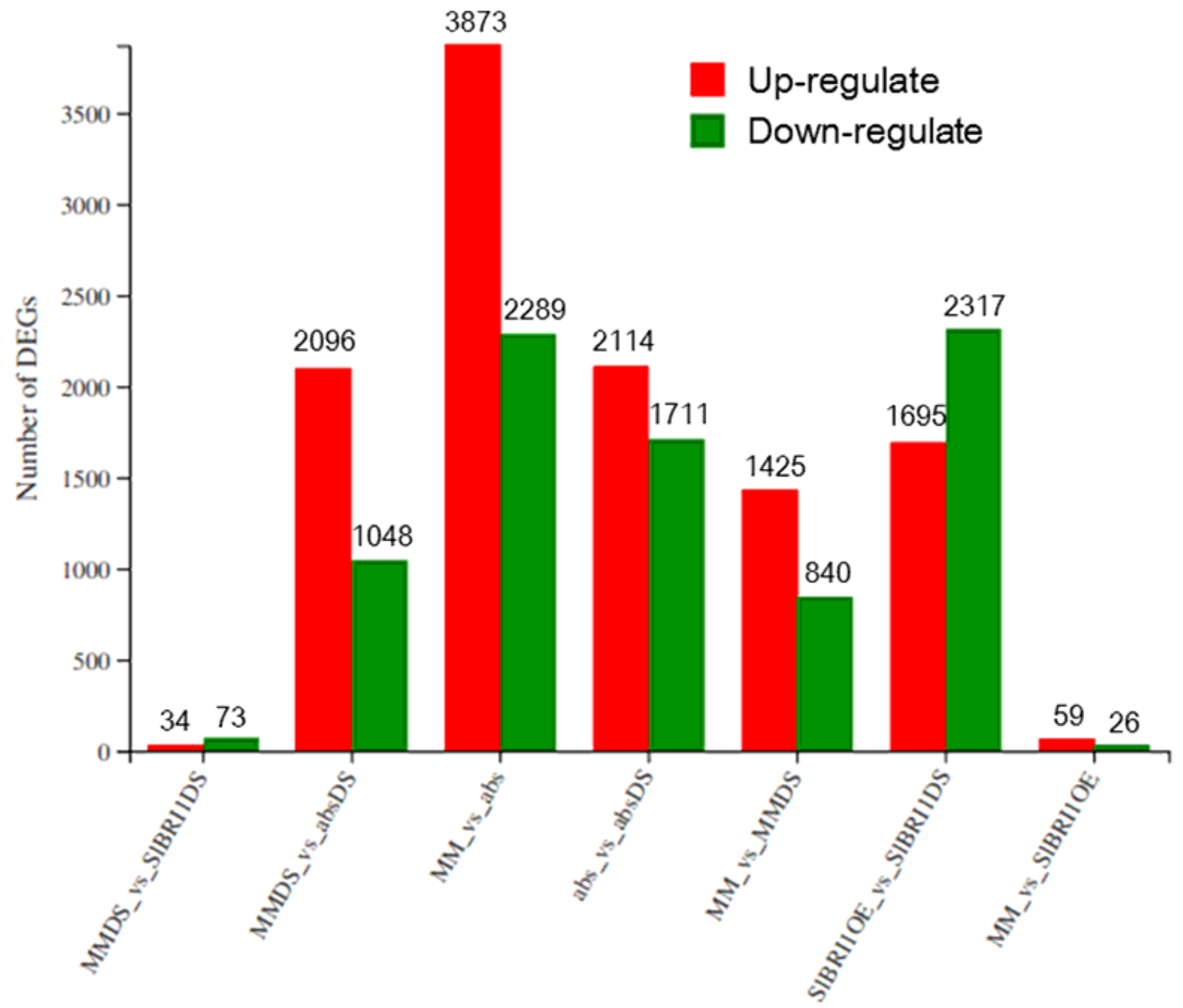

Figure 5

Number of DEGs among the seven comparison groups in tomato seedlings. 

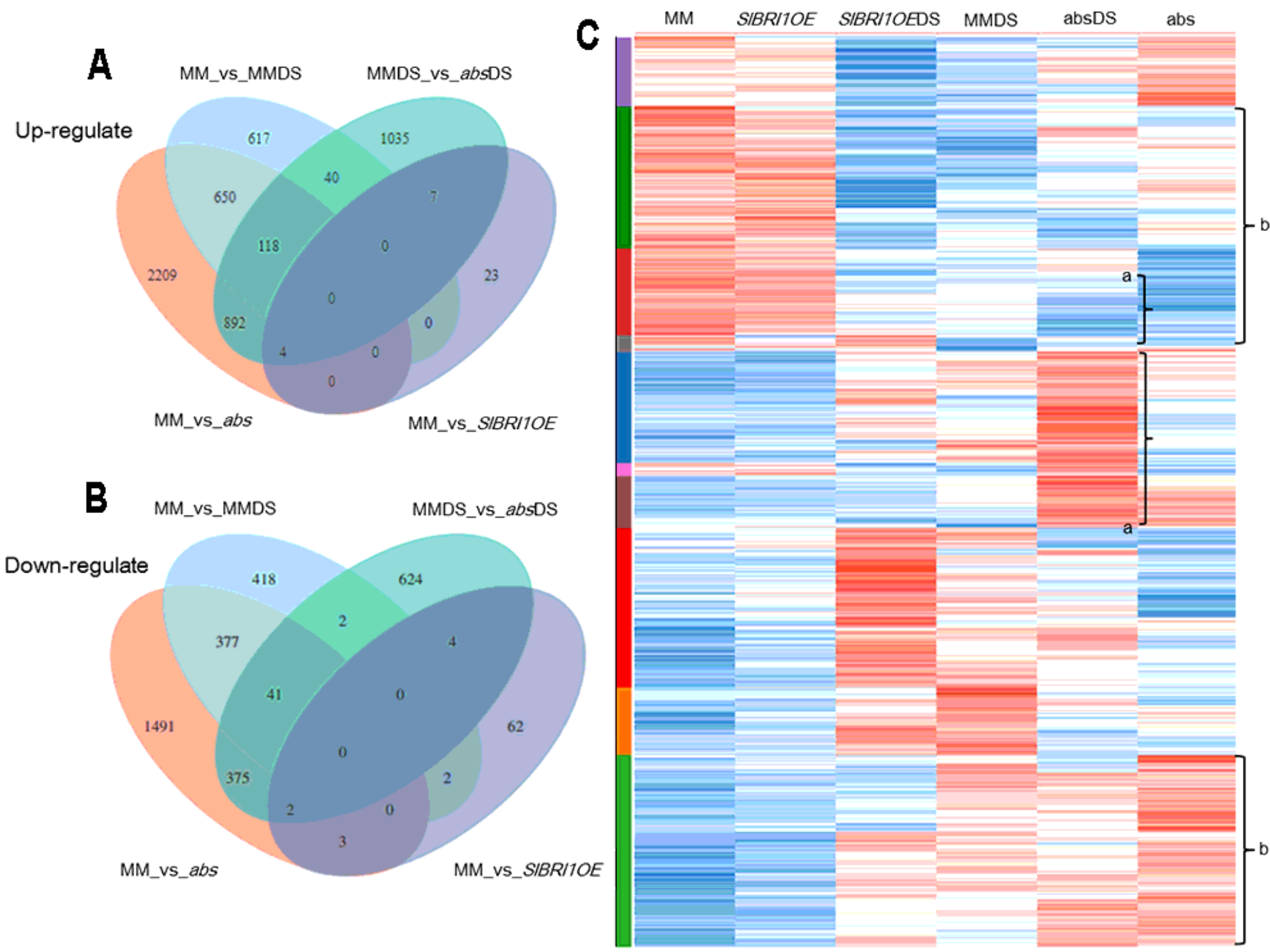

\section{Figure 6}

The number of DEGs among the four comparison groups in the Venn diagram showed overlap of upregulated (A) and downregulated (B) DEGs in tomato seedlings. (C) Heatmap of DEGs in each sample. 


\section{GO annotations analysis}

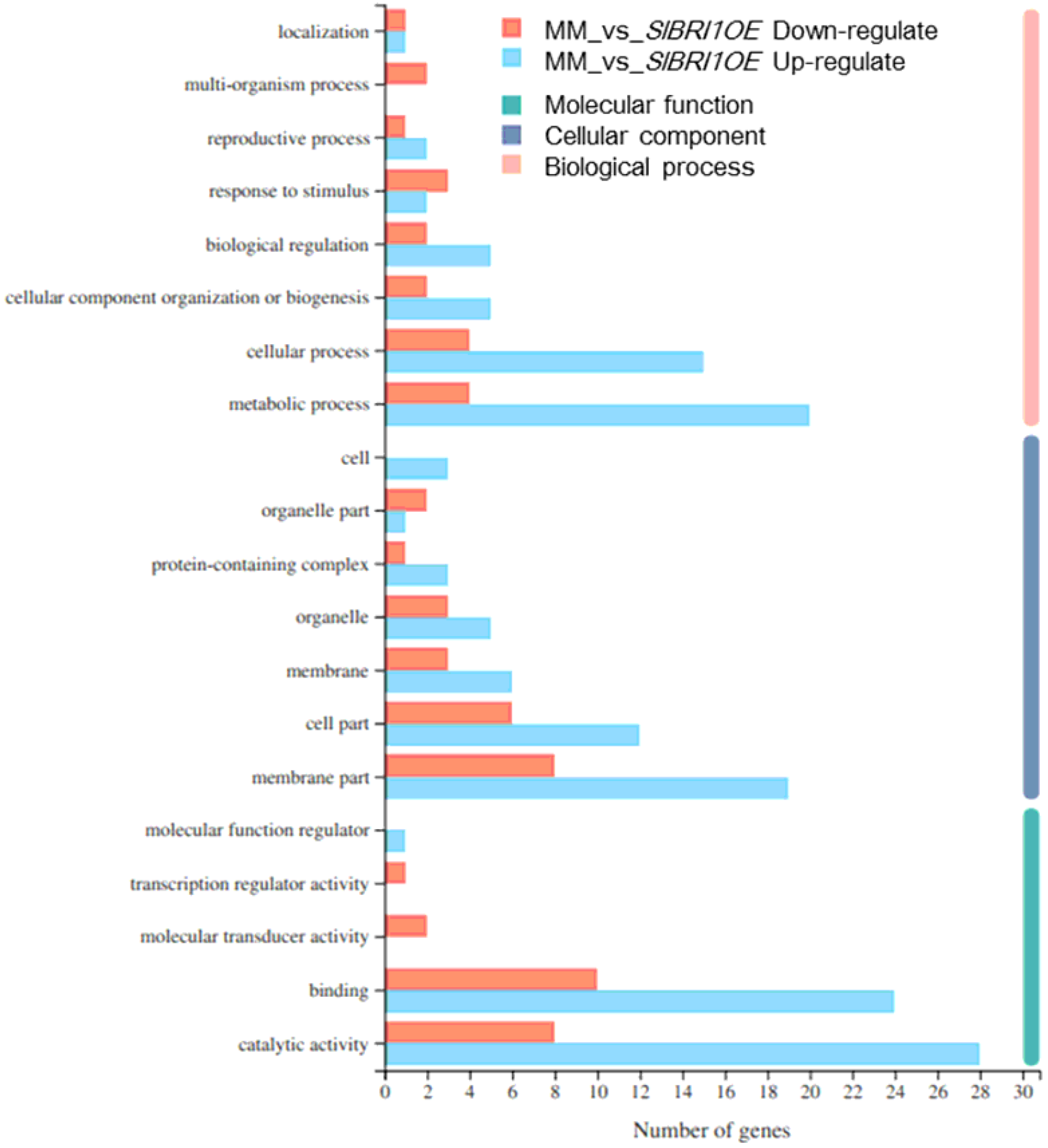

\section{Figure 7}

GO annotation analysis of DEGs between MM and SIBRI1OE. The x-axis indicates the number of genes in each classification, and the Y-axis indicates the GO classifications. 


\section{GO enrichment analysis (MM_vs_abs)}

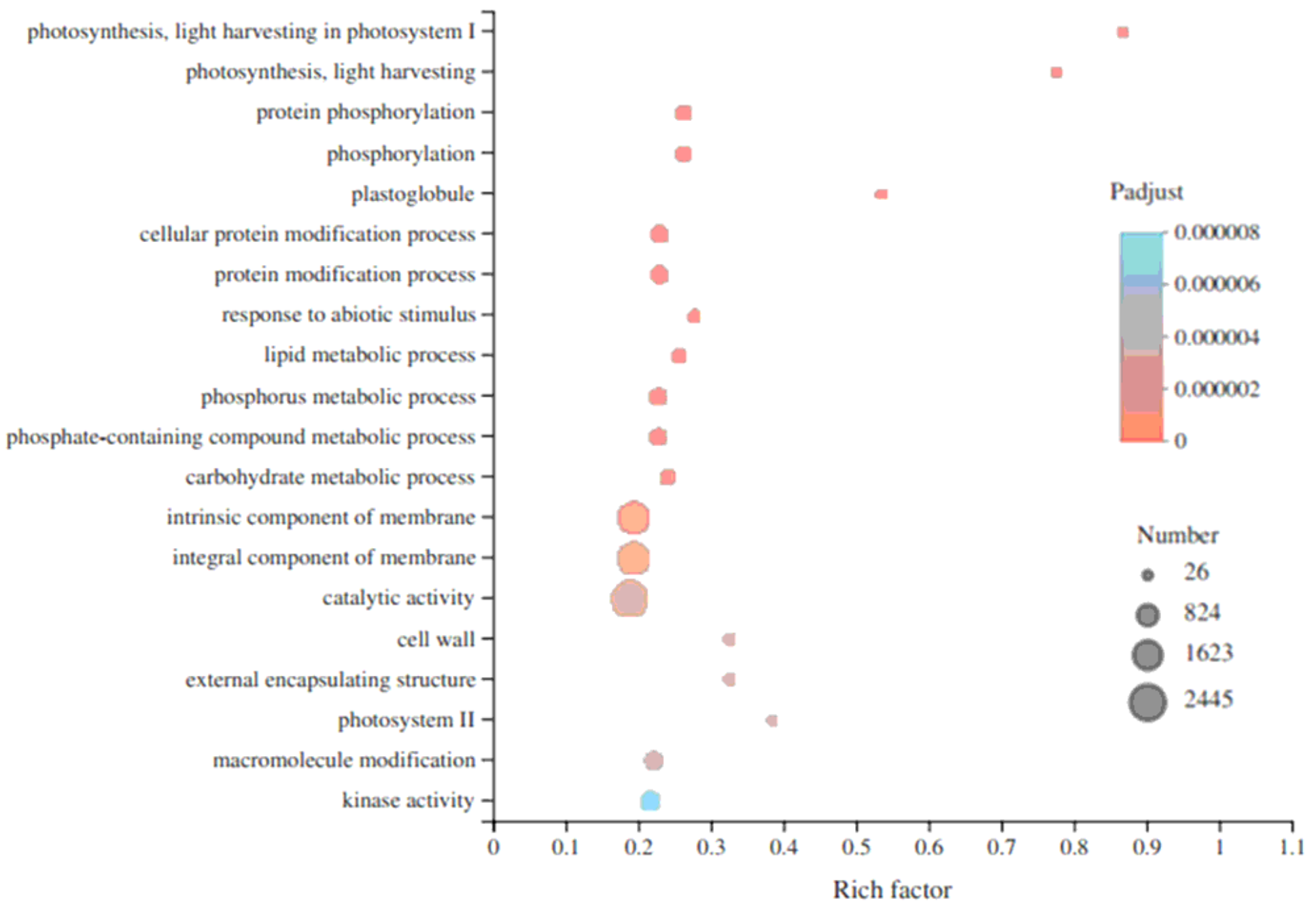

\section{Figure 8}

GO enrichment analysis of DEGs between the MM and abs. The X-axis shows the rich factor. Blue represents a high adjusted $P$ value, and red represents a low adjusted $P$ value. The $Y$-axis shows the top 20 KEGG pathways. The bigger size of spot, the more DEGs enriched. 


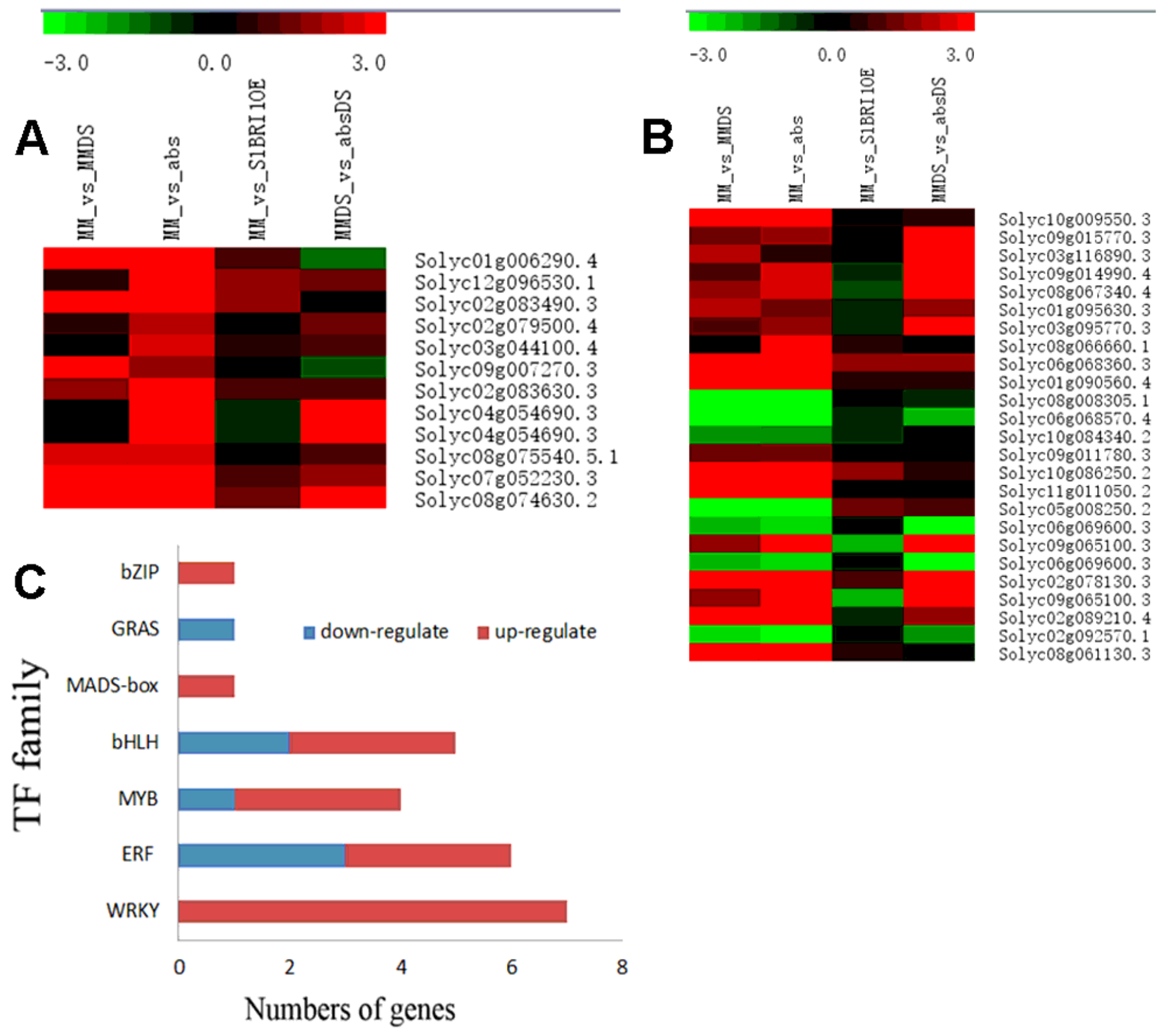

\section{Figure 9}

A heatmap heat map was constructed based on log2-transformed FPKM expression values of oxidoreductase activity genes (A) and each TF (B). (C) The numbers of differentially expressed TFs between MM and MMDS, MM and abs, MM and SIBRI10E, MMDS and absDS. 


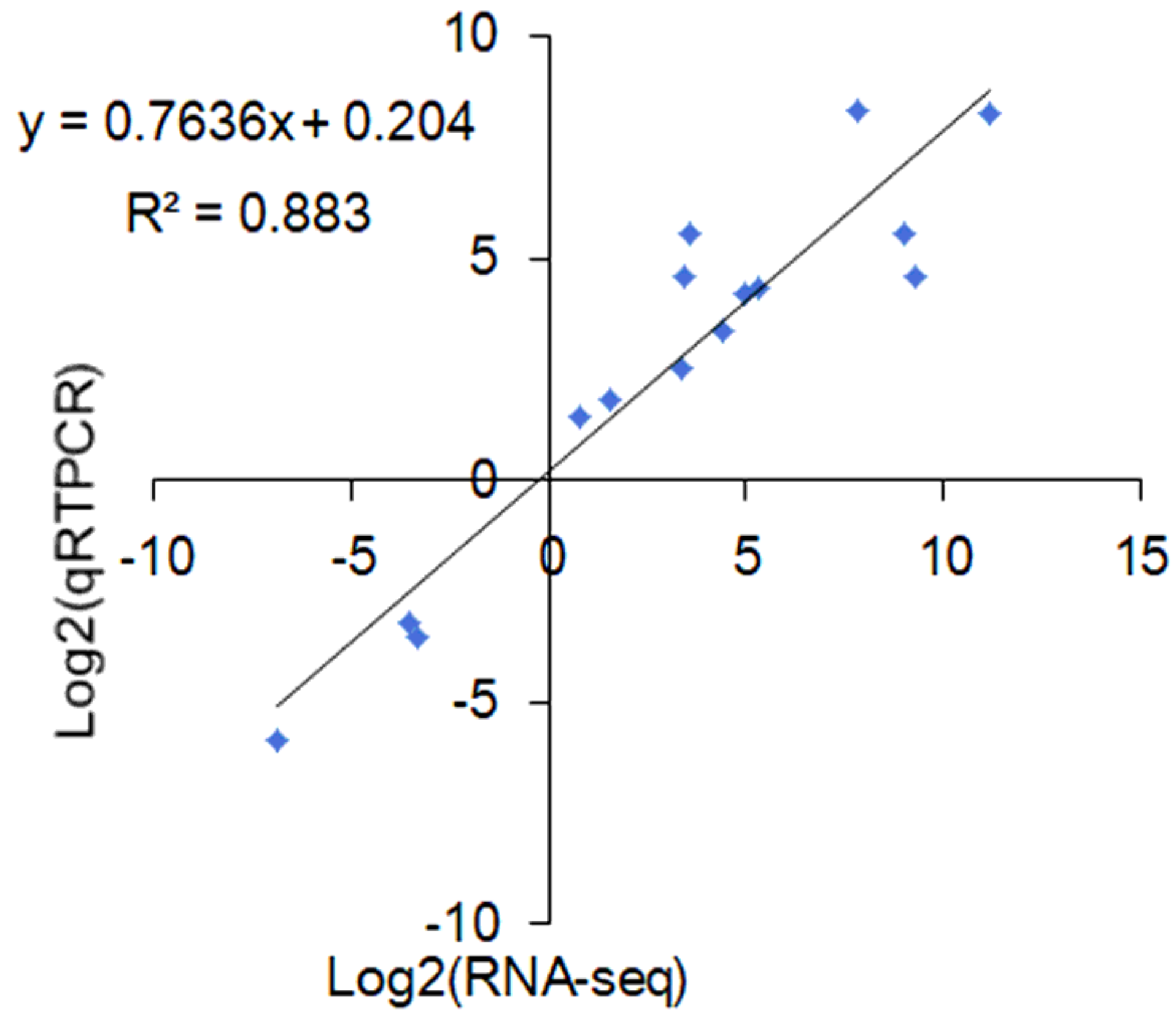

Figure 10

Validation of the RNA-Seq results by qRT-PCR. Data are presented as the means of three replicates.

\section{Supplementary Files}

This is a list of supplementary files associated with this preprint. Click to download.

- TableS1.docx

- Tables2.xIsx

- TableS3.xlsx

- Tables4.xls 
- Tables5.xls

- Tables6.xls

- Tables7.xls

Page 28/28 